GLOBAL SCATTERING MATRIX TECHNIQUE APPLIED TO THE CALCULATION OF HIGHER ORDER MODES FOR ILC SUPERCONDUCTING CAVITIES

I. Shinton and R.M. Jones; Cockcroft Institute, Daresbury; and The University of Manchester, UK 


\title{
GLOBAL SCATTERING MATRIX TECHNIQUE APPLIED TO THE CALCULATION OF HIGHER ORDER MODES FOR ILC SUPERCONDUCTING CAVITIES
}

\author{
I. Shinton and R.M. Jones; Cockcroft Institute, Daresbury; and The University of Manchester, UK
}

\begin{abstract}
A cascaded scattering matrix approach is used to determine the electromagnetic (e.m.) field in the main ILC cavities. This approach is used to compute higher order e.m. modes in the baseline configuration, and high gradient alternative configurations. We present results on three cavity designs: TESLA, Cornell University's reentrant and, KEK's "Ichiro". This approach allows realistic experimental errors to be incorporated in the studies in an efficient manner and allows several cavities to be modelled en masse. Simulations are presented on the wake-fields in super-structures and segments of entire modules. Details on trapped eigen-modes and means to avoid them are delineated.
\end{abstract}

\section{INTRODUCTION}

There is a need to be able to accurately model the higher order deflecting modes in the main linacs of the ILC en masse in which the effects of couplers, trapped modes and their influence on wake-fields and beam dynamics are ascertained. The necessity to be able to accurately predict the performance of the proposed ILC baseline design is further enhanced by the expected difficulties that will be present if alterations or cavity tuning is required within any given cryomodule. Even with a parallel code [1] meshing and accurately modelling the main linacs of the ILC will require vast resources and time. Moreover, the inclusion of realistic defects and misalignments into the baseline configuration will prove time consuming as it will potentially require remeshing of the problem. What we propose herein is a powerful technique that may be used to attempt to model a significant fraction of the main linacs in the ILC, the globalised scattering (S) matrix technique. The globalised scattering matrix technique is a relatively mature RF concept [2], in which the scattering matrix of a junction is obtained by "cascading" two sections at a time, these individual cascaded sections will henceforth be referred to as "unit cells". There are a number of methods that can be found throughout the literature, the form used in this paper is that of [2].

The generalised scattering matrix technique has been shown to be capable of accurately simulating structures [3]. The technique is very efficient and can readily incorporate misalignments and cavity perturbations into the calculation. Once the generalised cascaded matrix has been obtained for each cell in the structure in terms of every other cell in the structure the electromagnetic field (within an individual cell or a series of cells) can then be determined and trapped modes and other phenomena can be calculated. Here we present a preliminary demonstration of the usefulness of this technique applied to dipole modes within symmetric structures. Only dipole modes will be considered within this paper, because for a small offset in the beam and for small cell and cavity perturbations [4] these modes dominate the beam dynamics. The impact of these modes is described in terms of kick factors [5]. The distribution of these eigenmodes and kick factors are important as an adequate knowledge of them will allow beam dynamics simulations on the emittance of the beam down $11 \mathrm{~km}$ of the entire linac.

Unless otherwise specified in this paper all the $\mathrm{S}$ matrices required for the cascading calculations were carried out using HFSS v10; where a driven modal solution was sought in which the FEM mesh was adaptively refined until an overall accuracy below $0.01 \%$ was obtained for the resulting S parameters followed by a frequency sweep from 1 to $4.5 \mathrm{GHz}$ conducted in linear steps of $0.01 \mathrm{GHz}$. The dipole modes for the symmetrical structures calculated in this paper were modelled using a quarter of the geometry and $\mathrm{E}$ and $\mathrm{H}$ symmetry planes. All the $\mathrm{S}$ matrices, cascaded and plotted, were renormalized within HFSS using an impedance factor of $50 \mathrm{Ohms}$; in which the unit cell structure is taken from iris to iris. Finally, the finite conductivity of copper was used on all metallic surfaces to prevent numerical instabilities in the solutions [3].

\section{A Benchmarking Cascade Example}

As a benchmarking example of the cascaded matrix approach the test case of three joined TESLA [5] middle cells was investigated. Both a unit cell structure and a fully simulated structure were modelled. Both structures were modelled using six modes at each port. The cascaded three cell answer was directly compared to the fully simulated structure to gauge the accuracy of the technique; selected results of this are presented in Fig. 1. The cascaded results are in excellent agreement with those of the fully simulated structure with an average error of $0.3 \mathrm{MHz}$ which constitutes a percentage error of approximately $0.014 \%$. Not only are the cascaded results in very good agreement with the fully simulated results but the amount of computational time saved through cascading is considerable; the total time required to calculate the fully simulated structure was $29 \mathrm{hrs} 25 \mathrm{mins}$ and $20 \mathrm{sec}$, while the cascading calculation took $1.5 \mathrm{sec}$ to run on a single processor machine. (To obtain the total calculation time, the time to accurately compute a single cell must be added to this latter time). It may be noted that below the cut-off frequency for the $\mathrm{TE}_{11}$ mode (i.e. 2.5099 
$\mathrm{GHz}$ ) there are two peaks that do not fully match; this can be remedied by the inclusion of more modes and a finer mesh, but for the present discussion and the results presented hereafter it is a minor point because the inclusion of TTF beam pipes [5], in later simulations, will remove modes below the cut-off frequency.

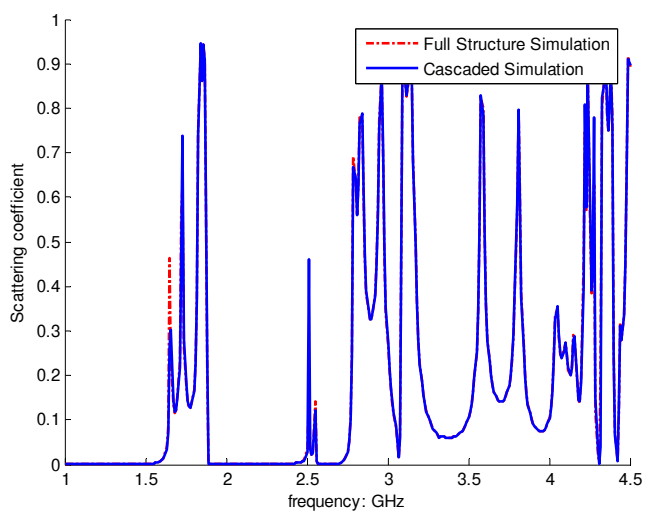

Figure 1: Comparison between the cascaded and fully simulated structure of the $\mathrm{S}_{21}$ matrix for the incident $\mathrm{TE}_{11}$ mode scattered into the $\mathrm{TE}_{11}$ mode.

\section{CASCADED SIMULATIONS}

\section{Tesla, Cornell and Ichiro Comparison}

The cavity designs of the TESLA [5], Cornell University's re-entrant [6] and KEK's "Ichiro" [7] were investigated using the cascading technique. Unfortunately during the period at which this preliminary research was conducted information and schematics relating to the beam pipes of KEK's "Ichiro" design were unavailable, so a complete study and comparison of cascaded coupled cavities was not possible. Here we present a direct comparison of the dipole HOM's of seven cascaded middle cells of the three designs. Fig. 2 shows the generalised cascaded $\mathrm{S}_{21}$ matrix component (for the incident $\mathrm{TE}_{11}$ mode scattered into the $\mathrm{TE}_{11}$ mode) for the fourth cell in the structure. The unit cell calculations were carried out using a total of six modes at each port.

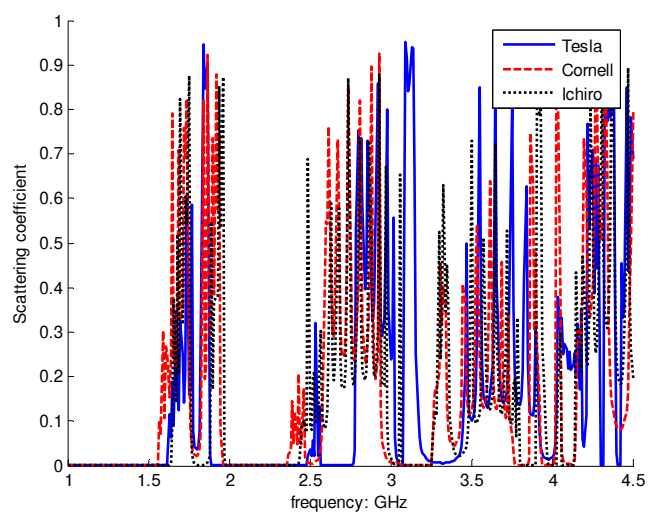

Figure 2: Comparison plot of the generalised cascaded $\mathrm{S}_{21}$ matrix component of the fourth cell in a 7 middle cell structure for the incident $\mathrm{TE}_{11}$ mode scattered into the $\mathrm{TE}_{11}$ mode of the three cavity designs.

\section{Cascaded Simulation of an Entire 72 Cell TESLA Module}

The unit cell calculations were carried out with a total of six modes at each port. The unit cells were simulated using information from [5] in which the TTF beam pipe configuration was used. Results from this calculation are presented in Fig. 3.

The generated results can then be used to investigate trapped modes in the superstructure, either by looking at individual vector plots of the resulting electromagnetic fields within every cell in the structure as seen in [1] or by a simpler method such as superimposing the cascaded results [8]. An efficient means of displaying the modes entails overlaying all transmission $S_{21}$ matrices, as illustrated in Fig. 3; trapped modes occur when the peak associated with modal resonances disappear.. Although this does not provide information as to location of the trapped mode, it does provide an efficient means of investigating the presence of trapped modes. The location of trapped modes in terms of this research remains the aspect of future work.

A possible means of eliminating the trapped modes of fields which do not penetrate the regions occupied by the HOM couplers is to purposely deform a cell in the region of the trapped mode to shift the cell frequency.

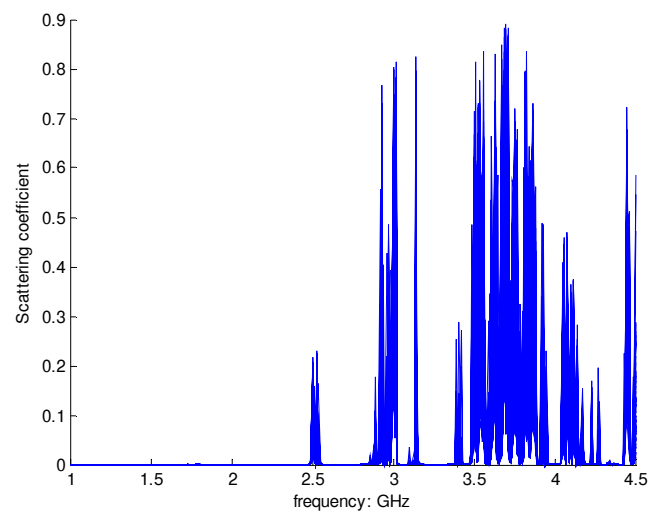

Figure 3: Overlay of the cascaded $S_{21}$ matrix for the incident $\mathrm{TE}_{11}$ mode scattered into the $\mathrm{TE}_{11}$ mode of each of the 72 cells in a complete TESLA module.

\section{DENTED STRUCTURE SIMULATION}

An initial investigation into the effect of cavity perturbations was investigated to gauge the sensitivity of a superconductive cavity to fabrication errors. A hemispherical dent placed on the equator of a TESLA cavity was simulated. As a consequence of the inherent asymmetry in the dented cavity, the entire cavity must be modelled. At least 10 modes at each port had to be considered, in order to take into consideration the dipole, quadrupole and sextupole HOM's that would be present (as there would be at least two preferred orientations for the possible port solutions to take). In order to accurately model the dent, a virtual object was created around the region of the dent into which a very fine mesh was introduced. The meshed virtual object can be observed in 
Fig. 4 below; in the area of the dent 5000 elements per cubic $\mathrm{mm}$ were introduced as well as surface refining. Unfortunately due to memory restraints on the $32 \mathrm{bit}$ machine on which this simulation was conducted only an accuracy of $0.01 \%$ could be obtained for the resulting $\mathrm{S}$ parameters.
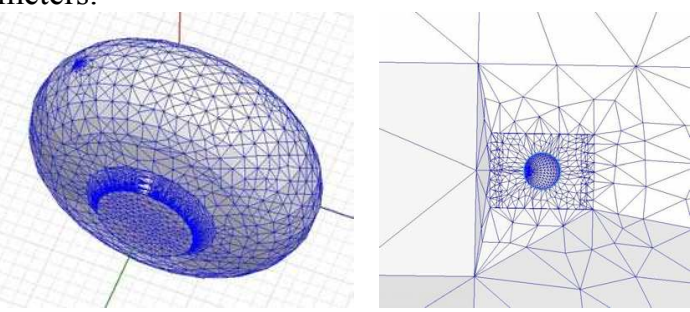

Figure 4: Final adapted mesh used to simulate a hemispherical dent with a radius of $1 \mathrm{~mm}$ on the equator of a middle TESLA cell. On the left the overall mesh. On the right a zoomed in inspection of the mesh used to model the dent.

A frequency scan between 1.7 to $2 \mathrm{GHz}$ was conducted in linear steps of $1 \mathrm{MHz}$ to identify the second dipole band within the TELSA cavity [5], this was followed by a more refined sweep conducted around the area at which mode degeneracy was split. A direct comparison between the fully simulated and dented cavity, illustrated in Fig. 5, revealed small frequency shifts. These results are preliminary, but nonetheless indicate splitting of the mode degeneracy and are of the same order of magnitude as observed experimentally [4]; other numerical simulations due to cavity deformation have also reported this phenomena [9].

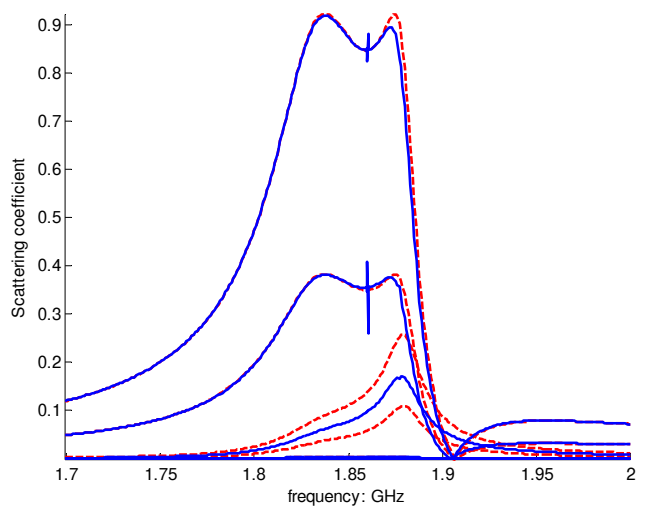

Figure 5: $\mathrm{S}_{21}$ matrix comparison between dented (blue line) and un-dented (dashed red line) fully simulated structure for scattering into the $\mathrm{TE}_{11}$ mode from all other six available modes.

\section{WAKEFIELDS AND FUTURE WORK}

Once the cascaded generalised scattering matrix has been derived for each cell in the simulated structure it is then possible to obtain the electromagnetic field within any cell in the structure by running another FEM solution for each cell in which the scattering matrix values are used as boundary conditions at each port. Most electromagnetic solver packages, such as HFSS, have the ability to use input $\mathrm{S}$ matrices to calculate the electromagnetic field in a user defined object. It then becomes a simple matter of using the standard formula [5] to calculate kick factors, a sum wake-field or more complex phenomena as seen for example in [8]. This remains an aspect of future work as does the extension of the generalised scattering matrix method to include couplers in the designs and simulations in which realistic machine and perturbations have been incorporated, with the final aim of the research to fully model the ILC with realistic effects and locate potentially harmful trapped modes.

\section{DISCUSSION}

As has been demonstrated the generalised scattering technique is a practical and useful method for obtaining the HOM's in an accelerating structure giving results which are comparable to those of a fully simulated structure requiring only a very small fraction of the resources and time. The three superconductive cavity designs have all been designed to operate at $1.3 \mathrm{GHz}$ in order to accelerate at high gradients the monopole mode. It is important to ensure that the HOMs are properly damped in order to avoid beam break-up instabilities or severe emittance dilution. For the three designs there is a significant redistribution of the modes, clearly the damping will need to be designed appropriately for each cavity structure.

The use of a generalised scattering technique to attempt to model the entire ILC is a simple yet practical way in which the problem may be attempted. The technique also has the added bonus of being able to easily incorporate realistic machining and alignment errors into the simulation without the necessity to "remesh" the entire geometry. In this way the method is capable of being able to quickly and efficiently generate realistic results. All the items presented in this paper are only preliminary in nature and remain the aspect of future work.

\section{REFERENCES}

[1] C. Ng et al., SLAC-PUB-12020, 2006.

[2] G.L. James, IEEE Trans MTT-29 10 (1981) 1059.

[3] R.M. Jones et al., PAC2003, p.1270, 2003.

[4] S. Molloy et al., Phys Rev Special Topics Accelerators and Beams 9 (2006) 112802.

[5] R. Wanzenberg, TESLA 2001-33, 2001.

[6] V. Shemelin and H. Padamsee, SRF0050808-06, 2006.

[7] K. Saito et al., KEK private communication.

[8] M. Dohlus et al., PAC1997, p. 2553, 1997.

[9] Lie-Quan Lee et al., SLAC-PUB-12141, 2006. 